\title{
Philosophiques
}

\section{L'éternel comme tiers synthétisant chez Kierkegaard}

\section{Maurice Carignan}

Volume 8, numéro 1, avril 1981

URI : https://id.erudit.org/iderudit/203150ar

DOI : https://doi.org/10.7202/203150ar

Aller au sommaire du numéro

Éditeur(s)

Société de philosophie du Québec

ISSN

0316-2923 (imprimé)

1492-1391 (numérique)

Découvrir la revue

Citer cet article

Carignan, M. (1981). L'éternel comme tiers synthétisant chez Kierkegaard.

Philosophiques, 8(1), 75-92. https://doi.org/10.7202/203150ar d'utilisation que vous pouvez consulter en ligne.

https://apropos.erudit.org/fr/usagers/politique-dutilisation/ 


\title{
L'ÉTERNEL COMME TIERS SYNTHÉTISANT CHEZ KIERKEGAARD
}

\author{
par Maurice Carignan
}

En 1852, alors que ses ouvrages devenaient de plus en plus polémiques et s'engageaient résolument dans une douloureuse lutte contre la chrétienté établie de son pays ${ }^{1}$, Kierkegaard écrivait dans son Journal:

L'homme est une synthèse, et donc naturellement pour ainsi dire un hypocrite né ou un être congénitalement pourvu de la possibilité d'être un hypocrite. Or la question posée par Dieu à chaque individu est: Seras-tu un hypocrite ou demeureras-tu en relation à la vérité ${ }^{2}$ ?

De par les éléments polaires qui le constituent comme synthèse, l'homme est à la fois spirituel et sensuel. Le moi spirituel donne un sens infiniment élevé à cette joie dont la bonne nouvelle du christianisme est imprégnée. Mais le moi sensuel avec lequel il est en tension interprète de façon glissante cette joie, et dans la mesure où il réussit à s'imposer, l'hypocrisie triomphe ${ }^{3}$.

Un autre passage du Journal, écrit deux ans plus tard en 1854, revient sur la même idée:

L'homme est une synthèse, un composé de plus bas et de plus haut, et depuis sa naissance, il est presque à la merci du plus bas . . Le christianisme convient à la partie plus élevée de la nature humaine, mais de façon à faire souffrir la partie plus basse ${ }^{4}$.

1. Kierkegaard venait de rédiger, sous le titre Jugez vous-mêmes, un ouvrage trop virulent pour être publié dès lors. Il y attaquait avec force l'Église officielle du Danemark et son plus haut dignitaire ecclésiastique, l'évêque Mynster.

2. Papirer, $\mathrm{X}^{4}$ 638. Notre traduction. Chaque fois que ce sera possible, nous citerons le Journal de Kierkegaard d'après la traduction et la présentation sélective Knud Ferlov et Jean-J. Gateau, éditée par Gallimard dans la collection «Les Essais».

3. Ibid.

4. Pap. X $1^{1} \mathrm{~A} 475$. Notre traduction. 
Bien sûr, ces passages, tout comme quelques autres du Journal où il est question de synthèse humaine ${ }^{5}$, renvoient implicitement au Concept d'Angoisse de 1844. Le pseudonyme Vigilius Haufniensis y affirme que l'homme est

une synthèse d'âme et de corps, mais... en même temps une synthèse du temporel et de l'éternel ${ }^{6}$.

Ils réferent également à La Maladie à la Mort de 1849 où Anti-Climacus renchérit:

L'homme est une synthèse d'infini et de fini, de temporel et d'éternel, de liberté et de nécessité...?

Si Kirkegaard et ses pseudonymes expriment de façon dialectique ce qu'est l'homme, c'est en réaction contre une notion substantialiste dans laquelle la pensée philosophique moderne s'était enlisée, minimisant par le fait même l'essentielle originalité du moi authentique en perpétuelle mouvance d'autoréalisation $^{8}$. Du fait qu'il est à la fois et indissolublement corps et âme,

5. Pap. VIII ${ }^{1}$ A 312, X ${ }^{4}$ A 482, X $1^{1}$ A 482, X $1^{1} \mathrm{~A} 408,592, \mathrm{X}^{2} \mathrm{~A} 246$.

6. Le Concept d'Angoisse, Oeuvres Completes V11, p. 185; Samlede Vaerker IV, p. 391. Nous emploierons les sigles $0 . C$. et $S . V$. pour signifier respectivement les Oetures Completes de Kierkegaard, édition de l'Orante, et la deuxième édition des Samlede Vaerker, udg. af A.B. Drachmann, J.L. Heiberg og H.O. Lange, Gyldendalske Boghandel, Kjobenhann, 1920-36.

7. La Maladie à la Mort, O.C. XVI, p. 171 ; S.V. XI, p. 143.

8. Notons ici à la fois un désaccord et un accord de Kierkegaard avec Hegel. Désaccord profond et insistant d'une part en ce qui concerne le sens mème de la dialectique. La dialectique hégélienne, aux yeux de Kierkegaard, est quantitative, s'exprimant dans l'abstraction logique de l'être vide, ". . . là tout est, et le tout n'est qu'unité et identité» (Pap. VII A 84). La dialectique kierkegaardienne, au contraire, est qualitative et exprime le devenir du moi dans le choix de la liberté (Voir Pap. I A 72, 75; IV A 192; VII A 80, 84, 191). Mais quand il s'agit du dynamisme qui définit essentiellement le moi, Kierkegaard s'inspire visiblement d'un passage de la Préface de la Phénoménologie de l'Esprit où Hegel dit du Moi-sujet qu'il est «but actualisé» (ausgefübrte Zweck), devenir procédant à son déploiernent; que «cette inquiétude (Unrube) est proprement le Moim... (Voir Phänomenologie des Geistes, éd. Johannes Hoffmeister, Hamburg: Felix Meiner, 1952, p. 22. Traduction française de Jean Hyppolite, La Phénoménologie de l'Esprit, Aubier, Éditions Montaigne, p. 20-21). Le texte hermétique de La Maladie à la Mort, où Anti-Climacus définit le moi (O.C. XVI, p. 171; S.V. XI, 143), s'éclaire considérablement quand on le compare à celui de Hegel, ce qu'a bien vu S. Crites (In the Twilight of Christendom: Hegel vs. Kierkegaard on Faith and History, Chambersburg Pa, 1971). Mark C. Taylor, dans son Kierkegaard's Pseudonymous Authorship, Princeton University Press, 1975, donne plusieurs citations pertinentes de la Phénoménologie où Hegel déclare expressément son intention de briser avec une notion substantialiste du sujet (Voir p. 102-108). Il (Taylor) montre en outre l'évolution de la notion de Substance à travers les philosophies de Descartes, Spinoza, Locke et Berkeley, jusqu’à son rejet radical par Hume pour qui les moi ne sont que des ubundles of sensations" (Taylor, ibid., p. 94-101). Bien que le passage de la Phénoménologie, dont Anti-Climacus semble bien s'être inspiré, traite du Moi absolu plutôt que du moi individuel, il reste que pour l'auteur de La Maladie à la Mort le moi (individuel) est aussi mouvance, inquiétudo essentielle plutôt que substance ou nature statique. On verra mème que l'idéal du vrai chrétien consiste pour Kierkegaard à vaincre une certaine substantialité illusoire synonyme de repos dans l'inertie. 
fini et infini, possibilité et nécessité, temporalité et éternité, l'homme recèle dans son être profond le ferment de ce qu'il sera dès lors que son moi idéal se sera rapporté à son moi réel. Ou, pour mieux dire, le moi est essentiellement cette tension même entre l'idéalité et la réalité.

Ce cheminement pénible vers l'actualisation du moi est identique, pour Kierkegaard et son pseudonyme Anti-Climacus, à celui par lequel on devient chrétien ${ }^{9}$. Il en va du christianisme incarné comme du moi, de l'esprit: il est essentiellement devenir, élan, mouvance sans repos, et cela sous peine d'être mensonger, illusoire, hypocrite.

Il n'est pas facile de faire l'exégèse des textes dans lesquels les pseudonymes Vigilius Haufniensis, Johannes Climacus et AntiClimacus définissent l'homme. Il faut reconnaitre le mérite des commentateurs qui s'y attachent ${ }^{10}$.

J'aimerais ici revenir sur une difficulté d'interprétation soulevée courageusement par l'un d'entre eux, Mark C. Taylor, dans son intéressant ouvrage Kierkegaard's Pseudonymous Authorsbip ${ }^{11}$.

Vigilius Haufniensis, ayant déterminé deux couples polaires qui définissent l'homme (corps-âme, temporel-éternel), déclare:

La synthèse du temporel et de l'éternel n'est pas une nouvelle synthèse; elle est une traduction de la première selon laquelle

9. Anti-Climacus dit en effet: «La foi consiste en ce que le moi, étant lui-même et voulant l'être, devient transparent et se fonde en Dieu» (La Maladie à la Mort, O.C. XVI p. 238; S.V. XI, P. 219).

10. Parmi les plus récents de langues française et anglaise, mentionnons André Clair, "L'homme malade et la santé comme tâche, selon Kierkegaard", dans Rev. Sc. Pbil. Théol., (LIV) 1970, p. 489-515 et 619-635; Pseudonymie et Paradoxe, Paris, Vrin, 1976; Mark C. Taylor, "Kierkegaard on the Structure of Selfhood", dans Kierkegaardiana, (IX) 1974, p. 84-101; et Op. cit., p. 81-126; John W. Eltod, Being and Existence in Kierkegaard's Psendonymous Works, Princeton University Press, 1975. Il convient aussi de mentionner les articies, moins récents, de Robert S. Hartman, "The Self in Kierkegaard", dans Journal of Existential Psychiatry, II (1962) p. 409-436; Louis Dupré, "The Constitution of the Self in Kierkegaard's Philosophy", dans International Pbilosophical Quarterly, III, (1963), p. 506-526; Paul Dietrichson, "Kierkegaard's Concept of the Self", dans Inquiry, VIII (1965), p. 1-32; et Jacques Colette, "Le désir d'être soi et la fonction du Père chez Kierkegaard", dans L'Inconscient, $\mathrm{n}^{\circ} 5$, janvier-mars 1968, p. 131-155.

11. Nous référons au chapitre trois de cet ouvrage, p. 81-126. 
l'homme est une synthèse d'âme et de corps portée par l'esprit. Dès que l'esprit est posé, l'instant est donné ${ }^{12}$.

À première vue, ce passage semble affirmer un strict parallélisme et même une identité réelle des deux synthèses: l'une, celle dont les éléments composants sont le corps et l'âme, s'accomplit dans l'esprit qui est le tiers synthétisant; l'autre réalise dans l'instant la jonction existentielle du temporel et de l'éternel. Le mot Udtrykket (la traduction, l'expression), employé par Vigilius pour caractériser le rapport entre les deux synthèses, semble évoquer une équivalence entre le corps et le temporel, l'âme et l'éternel, l'esprit et l'instant. Et ce parallélisme vaudrait également pour les deux autres couples dialectiques indiqués plus tard par Anti-Climacus: fini — infini, et nécessité - possibilité dont les éléments synthétisants sont respectivement le moi et la liberté $^{13}$.

Cependant, c'est encore dans Le Concept d'Angoisse que l'on peut lire cette déclaration déroutante:

La synthèse du corps et de l'âme doit être posée par l'esprit, mais l'esprit est l'éternel, et par suite il n'est que lorsqu'il pose la première synthèse en même temps que la seconde, celle du temporel et de l'éternel ${ }^{14}$.

Mark C. Taylor montre très bien la difficulté d'un tel passage. L'esprit, tiers synthétisant des polarités corps-âme, est identifié à l'éternel qui, lui, est un élément polaire du couple temporel-éternel. Ce n'est pas l'âme que Vigilius identifie à l'éternel, mais plutôt le facteur même qui réalise la synthèse corps-âme, c'est-à-dire l'esprit. Ainsi, le parallélisme qu'une première déclaration de Vigilius semblait affirmer ne tiendrait plus: la synthèse du temporel et de l'éternel ne serait pas purement et simplement une traduction littérale de celle du corps et de l'âme. Du reste, le même auteur pseudonyme avait prévenu implicitement ses lecteurs contre un parallélisme aussi facile en disant:

Pour la seconde synthèse (i.e. celle du temporel et de l'éternel), il saute aux yeux qu'elle s'effectue autrement que la première. Dans

12. Le Concept d'Angoisse, O.C. VII, p. 188; S.V. IV, p. 395.

13. La Maladie à la Mort, O.C. XVI, p. 186; S.V. XI, p. 160.

14. Le Concept d'Angoisse, O.C. VII, p. 190; S.V. IV, p. 397. Les soulignés, localisant la difficulté elle-même d'interprétation, sont de nous. 
celle-ci, l'âme et le corps sont les deux moments et l'esprit est le tiers . . . La seconde synthèse n'a que deux moments: le temporel et l'éternel. Où est le tiers? S'il n'y en a pas, il n'y a pas proprement de synthèse, qui, étant une contradiction, ne peut s'achever comme synthèse que dans un tiers ${ }^{15}$.

La solution proposée par Taylor s'articule comme ceci: il faut prendre à la lettre les trois affirmations de Vigilius Haufniensis. Ainsi, il existerait bien un certain parallélisme entre la première et la seconde synthèse. Mais ce qui serait l'équivalent du temporel, ce serait le couple en tension dialectique des polarités corps et âme, elles-mêmes réellement identiques aux deux autres couples ajoutés par Anti-Climacus, fini et infini, nécessité et possibilité. Le tiers synthétisant de ces polarités équivalentes serait identique à l'éternel au sens analogique d'immobile, d'inchangeable, de constant. Telle serait la liberté, facteur de synthèse équivalant au moi et à l'esprit. Ainsi, de par la tension dialectique de ses éléments composants, l'homme serait établi dans sa temporalité synonyme de mouvance, d'inquiétude ontologique. Sa liberté (son moi, son esprit) serait ce tiers inchangeant ou éternel accomplissant la synthèse. L'éternel, identique à la liberté, à l'esprit ou au moi constituerait l'homme dans sa temporalité essentielle. Et l'instant serait ce lieu privilégié dans lequel le temps serait imprégné d'éternité, et l'éternité imprégnée de temporalité.

Si le moi et l'esprit peuvent s'identifier à l'éternel, c'est par le biais de la liberté. Déjà l'assesseur Wilhelm de L'Alternative, deuxième partie, n'avait-il pas affirmé cette identité du moi et de la liberté:

Mais qu'est-ce donc que le moi, ma personne? Si je voulais caractériser son premier instant, sa première expression, je répondrais: c'est la chose la plus arbitraire de toutes, et elle est néanmoins en même temps la plus concrète de toutes - c'est la liberté ${ }^{16}$.

Et Anti-Climacus, le chrétien extra-ordinaire de La Maladie à la Mort, fait écho aux dires de l'éthicien:

Le moi est composé d'infini et de fini. Mais cette synthèse est un rapport, et un rapport qui, bien que dérivé, se rapporte à luimême, ce qui est la liberté. Le moi est liberté ${ }^{17}$.

15. Ibid., O.C., VII, p. 185; S.V. IV, p. 391.

16. L'Alternative II, O.C. IV, p. 193; S.V. II, p. 231.

17. La Maladie à la Mort, O.C. XVI, p. 186; S.V. XI, p. 160. 
Cette éclairante solution ne va pas, me semble-t-il, sans une notion bien précise de la liberté qu'il faudrait expliciter. À cette fin, qu'on me permette d'abord de chercher le sens que Kierkegaard et ses pseudonymes concernés donnent à ce mot.

Dissertant sur "L'équilibre de l'esthétique et de l'éthique" au profit de son ami l'esthéticien A, (auteur de L'Alternative, première partie), l'assesseur Wilhelm remarque que, pour la philosophie, «il y a une médiation absolue», ce qui est très important pour elle, puisque "si l'on renonce à la médiation, on renonce à la spéculation». Mais une telle concession, se demandet-il, n'est-elle pas dangereuse? Ne conduit-elle pas logiquement à nier qu'il y ait un choix absolu, un dilemme absolu? Non, répond-il, si l'on a bien soin de ne pas confondre la sphère du raisonnement et celle de la liberté. Dans la première sphère, l'opposition ne perdure pas ... Elle est médiatisée et de là passe dans une unité supérieure. Mais dans la sphère de la liberté subsiste l'opposition, l'alternative dont un élément exclut l'autre. Et l'assesseur enchaîne:

Je ne confonds nullement le liberum arbitrium avec la vraie liberté positive; car même celle-ci tient de toute éternité le mal en dehors d'elle, bien que comme une impuissante possibilité, et elle ne s'echemine pas vers la perfection en accueillant le mal toujours plus, mais en l'excluant toujours davantage; mais l'exclusion est justement le contraire de la médiation ${ }^{18}$.

Ce passage révèle la conception que se fait l'éthicien du libre arbitre: contrairement à la vraie liberté qui affirme sa positivité en excluant le mal, le libre arbitre, ou encore la liberté d'indifférence, est de nature négative en ce que son choix porte sur l'alternative du bien ou du mal. Dans son premier jet des Miettes Philosophiques de 1844, Kierkegaard poursuivait dans la même ligne de pensée:

Le liberum arbitrium susceptible de choisir indifféremment le bien ou le mal est fondamentalement une abrogation du concept de liberté et un aveu d'échec à l'expliquer. Liberté signifie être capa-

18. L'Alternative II, O.C.' IV, p. 158; S.V. II, p. 188. 
ble. Le bien et le mal n'existent nulle part en dehors de la liberté puisque cette distinction n'apparaît que par la liberté ${ }^{19}$.

Les deur: textes comparés et combinés affirment que la vraie liberté, pour autant qu'elle existe, est antérieure au libre arbitre, qu'elle signifie une capacité dont le corrélatif est dans le sens de la perfection et exclut par le fait même le mal. Quiconque la réduirait au libre arbitre entendu au sens d'équilibre entre le bien et le mal ne ferait rien d'autre que la nier en l'avilissant.

Vigilius Haufniensis retrace l'origine psychologique de la liberté. Parlant du «génie religieux . . . qui refuse de s'en tenir à son immédiateté», il dit de lui qu' «en se tournant vers lui-même, il se tourne de ce fait vers Dieu» et se reconnaît coupable. La faute, voilà ce qu'il découvre en lui-même avec acuité. Mais de ce fait, il découvre aussi la liberté.

... la liberté (dit-il) fait sa félicité; non la liberté de suivre son bon plaisir dans le monde ... mais la liberté de savoir avec soi-même que l'on est la liberté 20 .

Pourtant, ce génie religieux, qui se délecte dans le fait d'être liberté, éprouve en même temps l'angoisse du péché, l'angoisse de cette faute possible qui pourrait lui ravir sa liberté. Et Vigilius enchaîne:

On voit que la liberté n'est nullement pouvoir de rébellion, ni liberté égoïste au sens fini, suivant une conception qui a souvent essayé d'expliquer l'origine du péché ... À concevoir ainsi la liberté, on place son contraire dans la nécessité... Mais le contraire de la liberté, c'est la faute ${ }^{21}$.

Autrement dit, on a maintes fois expliqué le péché originel par l'émergence de la liberté, entendant par ce mot la possibilité consciente de la désobéissance ou encore celle d'un choix irrémédiablement plafonné dans le fini. On sait avec quelle finesse et pénétration psychologique Vigilius avait montré le lien qui unit innocence, ignorance et angoisse, et comment celle-ci, vertige de la liberté, était la condition psychologique de cette rupture que fut le premier péché. «L'angoisse», avait-il dit, "est la réalité de la liberté comme possibilité offerte à la possibilité» 22 . En réalité,

19. Pap. VB56:2. Voir texte parallèle dans Le Concept d'Angoisse, O.C. VIII, p. 210; S.V. IV, p. 420.

20. Le Concept d'Angoisse, O.C. VIII, p. 206; S.V. IV, p. 415.

21. Ibid., O.C. VIII, p. 207; S.V. IV, p. 416.

22. Ibid., O.C. VIII, p. 144; S.V. IV, p. 346. 
il est impossible d'expliquer le passage de l'innocence à la faute: d'une part, le péché ne peut être entré dans le monde de façon nécessaire, et, d'autre part, il ne peut davantage sortir d'un libre arbitre abstrait ... ${ }^{23}$ Avant que ne se produise cette rupture, ce saut, la liberté n'est là que comme possibilité éveillée par l'angoisse. Dès lors:

Vouloir expliquer l'entrée du péché dans le monde d'une manière logique, c'est une sottise dont seuls peuvent s'aviser les gens hantés du ridicule souci d'une explication ${ }^{24}$.

Vigilius veut faire ressortir cette idée que la vraie liberté n'est pas, par essence même, aux antipodes du nécessaire. Tel serait ce qu'on appelle communément le libre arbitre qui, ainsi conçu, n'existe tout simplement pas ${ }^{25}$. La vraie liberté n'a comme contraire que la faute. Elle dit essentiellement indépendance, absence d'esclavage ou de lien vis-à-vis de la faute. L'homme libre, en ce sens, n'est pas celui qui aurait indéfiniment et invariablement un pouvoir de choisir entre telle ou telle ligne d'action, mais plutôt celui qui d'ores et déjà se serait donné à lui-même une seconde nature le prémunissant contre la faute. Le prototype de l'homme libre, le "génie religieux», ce serait l'ascète qui aurait lui-même bâti sur sa facticité une orientation l'éloignant au maximum de la possibilité de sombrer dans la faute. «Se choisir soi-même», pour revenir à une expression de l'éthicien Wilhelm, c'est briser le cercle des choix masqués pour intégrer la sphère de la «validité éternelle» où l'on apprend à choisir avec une certaine nécessité croissante ce dont la contradictoire est la faute.

Kierkegaard lui-même confirme cette interprétation dans plusieurs passages de son Journal. Ainsi, une entrée de 1850 montre que le christianisme impose à l'homme un choix primordial, celui du royaume de Dieu, l'obligeant par le fait même à fuir cet état de complète indifférence que serait le libre arbitre. Et il poursuit:

Peut-on exprimer avec plus de précision que la liberté de choix n'est qu'une détermination formelle dans la liberté, et qu'accentuer la liberté de choix est la perte de la liberté? Le contenu de la

23. Ibid., O.C. VII, p. 151; S.V. IV, p. 354.

24. Ibid., O.C. VII, p. 151; S.V. IV, p. 354.

25. Ibid., O.C. VII, p. 151 et $210 ;$ S.V. IV, p. 354 et 420. 
liberté est à ce point décisif pour elle, que la liberté de choix n'a justement de vérité que parce qu'il ne faut pas de choix, encore qu'il $y$ en ait pourtant un ${ }^{26}$.

Le chrétien, sous peine de se nier lui-même, doit choisir l'unique nécessaire, le royaume de Dieu. «C'est cela», dit-il, «être esprit» ${ }^{27}$. Bien sûr, cette obligation serait en elle-même inintelligible si elle ne présupposait pas la possibilité physique d'une détermination contraire. Il est physiquement possible à l'homme de choisir la faute, c'est-à-dire le fini qui contredit l'option du royaume de Dieu. Mais, d'autre part, ce choix doit être éliminé et faire place à l'option primordiale qui signifie la vraie liberté. C'est malheureusement ce qu'en général on ne comprend pas.

. . . c'est parce que les hommes sont loin d'être esprit que la liberté leur donne tant de tracas, empêtrés qu'ils restent dans la liberté du choix. La réflexion ... en vient à s'hypnotiser sur la liberté de choix au lieu de se rappeler qu'il ne faut pas de choix... et ensuite choisir $^{28}$.

Si la vraie liberté tend vers l'exclusion du choix, c'est que le moi concret, l'esprit, est capable d'historicité. Ainsi, dit Kierkegaard en 1851:

Que c'est une illusion que ce libre arbitre; comme si à chaque instant de la vie on était toujours pourvu de cette possibilité abstraite, de façon à rester toujours au même point; comme si la liberté n'était pas en même temps un état historique ${ }^{29}$.

La liberté, en tant qu'identique au moi, à l'esprit, a une histoire, s'inscrit dans un devenir incessant sous peine de n'être pas. Par une comparaison très simple, Kierkegaard explique ce qu'il faut entendre par là. À cause de la délibération, de l'arbitrage rationnel impliqué dans l'acte de choisir une ligne d'action, le libre arbitre peut se comparer à une balance. Prenons, dit-il, la plus fine de toutes, celle dont on se sert pour peser l'or. Son usage répété constitue une «histoire», une tendance toujours plus accusée, par exemple, à pencher d'un côté ou de l'autre. Et Kierkegaard continue:

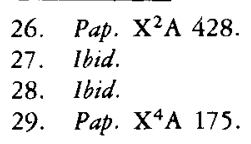


De même pour la volonté. Elle a une histoire qui ne cesse de courir. Et ça peut aller jusqu'au point que l'homme finisse par perdre la faculté de choisir ${ }^{30}$.

Dans le cas, an-historique, de celui qui ne s'étant jamais choisi lui-même s'est toujours ancré davantage dans la faute en confinant ses «choix» à la sphère du fini, un tel état d'esclavage «est le châtiment du péché ... ., et c'est encore du péché» ${ }^{31}$. C'est le cas de Don Juan dont la "liberté» n'est qu'indifférence et vanité. Par contre, celui qui a librement fait éclater cette sphère d'esthétisme, comme l'assesseur Wilhelm, s'engage par le fait même dans une histoire, dans la formation graduelle de son moi. Une nécessité se dessine en lui de choisir le général. S'étant choisi lui-même dans sa validité éternelle en fuyant ce qui est vain et éphémère, cet éthicien est libre. Pourtant, sa liberté ne sera vraiment en voie vers la perfection que lorsque ses choix se feront devant Dieu. Alors émergera une nécessité de se choisir comme individu conformément à sa vocation personnelle, distanciée comme il se doit de la séduction esthétique et du général éthique. La liberté, dit ailleurs Kierkegaard, "parcourt plusieurs stades avant de parvenir à elle-même» ${ }^{32}$. C'est là son histoire, celle d'une affirmation graduelle de la nécessité face au choix fondamental de soi-même comme individu (Enkelte). Un autre passage du Journal est encore plus explicite quant à l'affirmation du lien entre liberté et nécessité:

Oh! que de vérité et d'expérience dans ce que dit saint Augustin de la vraie liberté (différente de la liberté de choisir): le sentiment de liberté le plus fort qu'ait l'homme, c'est quand, définitivement résolu, il imprime à son action comme une frappe cette nécessité intérieure qui exclut l'idée d'une autre possibilité! Alors le «tourment» de la liberté ou du choix prend fin ${ }^{33}$.

Si maintenant nous résumons la position de Kierkegaard et de ses pseudonymes au sujet de la liberté, nous avons donc ceci: 1) la vraie liberté exclut la possibilité morale de choisir ce qui est mal, et ne se situe qu'au niveau positif de perfectionnement de l'homme. 2) À ce niveau, la vraie liberté tend à composer avec une certaine nécessité affectant la volonté historicisée. 3) Dès lors le

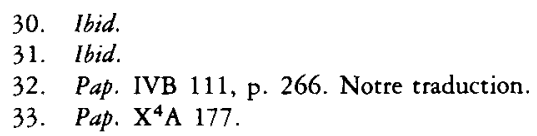


libre arbitre n'existe pas au sens d'équilibre, d'indifférence ou d'indétermination complète. 4) Par contre, entendu comme une capacité physique de choix, le libre arbitre existe, au mieux comme un grand bien, mais risque de battre en brèche la vraie liberté.

Étant donné que Kierkegaard ${ }^{34}$ et son pseudonyme Vigilius Haufniensis ${ }^{35}$ en appellent à l'autorité de Leibniz pour rejeter le libre arbitre ${ }^{36}$, on pourrait croire que leur position implicite est celle d'un étanche déterminisme psychologique ${ }^{37}$. On se tromperait alors, semble-t-il. Comment, en effet, comprendre les analyses de l'assesseur Wilhelm et de Vigilius Haufniensis sans présupposer que, pour eux, l'homme a la possibilité de choisir ... Du reste, l'entrée du Journal que nous venons de citer admet explicitement qu'une telle liberté existe comme un grand bien dont l'homme est doté:

En général, on la représente comme un bien extraordinaire, cette liberté de choisir. Et elle l'est aussi; mais cela dépend cependant aussi du temps qu'elle doit durer. Car on se trompe d'habitude à s'imaginer que c'est un bien précisément qu'elle dure toute notre $\mathrm{vie}^{38}$.

34. Pap. IVC 31, 36, 39

35. Le Concept d'Angoisse, O.C. VII, p. $151 ;$ S.V. IV, p. 420.

36. Comme le montre de façon convaincante un article de Nelly Viallaneix, Kierkegaard avait pour Leibniz une très grande admiration, le citant avec abondance dans les Papirer de novembre 1842 à mars 1844 et lui adressant même des louanges, ce qu'en général il ne fait pas souvent à l'endroit d'un philosophe! («Kierkegaard, lecteur de Leibniz», dans Critique (Revue générale des publications françaises et étrangères), oct. 1969, p. 895-914).

37. Leibniz avait écrit: "Tout est ... certain et déterminé par avance dans l'homme, comme partout ailleurs, et l'âme humaine est une espèce d'automate spirituel, quoique les actions contingentes en général, et les actions libres en particulier, ne soient point nécessaires pour cela d'une nécessité absolue, laquelle serait véritablement incompatible avec les contingentes" (Essais de Théodicée, première partie, $n^{\circ} 52$ ). Toute sa position sur la liberté est clairement formulée dans un fragment (Fragment $\mathrm{H}$ ): "Libertas indifferentiae est impossibilis. Adeo ut ne in Deum quidem cadat, nam determinatus ille est ad optimum efficiendum. Et creaturae semper ex rationibus internis externisque determinantur. - Quo plus substantiae sunt per se determinatae et ab indifferentia remotae, eo sunt perfectiores . . - - Eo major est libertas, quo magis agitur ex ratione, et eo major est servitus, quo magis agitur ex animi passionibus» (C.I. Gerhardt, Die philosophiscben Schriften von G.W. Leibniz, Bd VII, p. 109).

38. Pap. $\mathrm{X}^{4} \mathrm{~A}$ 177. Les soulignés sont de nous. 
$\mathrm{Au}$ point où nous en sommes, nous savons que pour les pseudonymes Vigilius Hautniensis et Anti-Climacus - et très vraisemblablement pour Kierkegaard lui-même --, les tiers synthétisant moi, esprit et liberté sont une seule et même réalité identique à l'éternel, élément d'une synthèse suprême réalisée dans l'instant. Nous savons également que, pour eux, la liberté implique des degrés d'autonomie ou d'indépendance dont le plus bas, - d'ailleurs susceptible de disparaître au profit du palier suprême, - est le libre arbitre ou la liberté de choix.

Le moment est venu d'examiner le sens que Taylor donne au mot liberté quand, dans son exégèse, il l'identifie au moi, à l'esprit, à l'éternel.

Reprenant la difficile affirmation d'Anti-Climacus à l'effet qu «Le moi est un rapport qui se rapporte à lui-même» ${ }^{39}$, il l'interprète, avec raison croyons-nous, comme signifiant: le moi est un rapport qui rapporte son moi idéal, potentiel, infini, à son moi réel, actuel, fini ${ }^{40}$. Cette interprétation a le grand mérite de bien marquer la tension, le dynamisme, le devenir qui, aux yeux de Kierkegaard et de ses pseudonymes, constituent l'essence même du moi. Rapporter son moi idéal à son moi réel, c'est entrer de plain-pied dans ce processus pénible par lequel on se donne à soi-même la perfection due à sa propre nature d'homme.

Bien sûr, cette activité du moi se bâtissant lui-même ne va pas sans l'exercice du libre arbitre, de la liberté de choix. À vrai dire, on ne saurait s'expliquer que le moi puisse transmuer l'idéalité en la réalité sans avoir à poser le choix fondamental de soi-même, à choisir le choix au lieu de tendre, comme l'esthéticien $A$, vers l'impossible abstention radicale ${ }^{41}$. Mais pour définir

39. La Maladie à la Mort, O.C. XV1, p. 171; S.V. XI, p. 143.

40. Mark C. Taylor, op. cit., p. 117.

41. Voir "Ou bien - Ou bien, Discours d'un extatique", dans L'Alternative I, O.C. III, p. 39 ss.; S.V. I, p. 26 ss. L'alternative devant laquelle se trouve l'esthéticien est un véritable dilemme. Que l'on choisisse ceci ou cela, de toute façon ou regrettera d'avoir ainsi choisi. Dès lors, l'option de l'esthéticien A est de se retirer dans cette éternité de l'imagination qui réconcilie dans l'indifférence les éléments du dilemne. Mais il s'agit là, au fond, d'une vaine masquarade où l'esthéticien prétend camoufler indéfiniment son non-engagement. L'assesseur Wilhelm dénoncera cette vanité et inconséquence de son ami $A ;$ « . . . ne sais-tu pas que vient l'heure de minuit ou chacun doit lever le masque; crois-tu que la vie entend toujours raillerie; crois-tu qu'on peut se faufiler un peu avant les douze coups pour éviter ce moment?" (L'Alternative II, O.C. IV, p. 145-146; S.V. II, p. 174). 
le sens précis de cette liberté qui s'identifie à l'éternel, il faut se rapporter, comme le fait Taylor, à la signification que revêt ce dernier terme: éternel.

On ne saurait prétendre que l'éternité du moi ou de l'esprit soit celle même de Dieu. Kierkegaard et ses pseudonymes ont trop le sens de la transcendance divine pour qu'on puisse leur prêter une telle conception. Il faut ici comprendre ce terme de façon analogique. Or l'inchangeable, l'immobile peut se dire analogiquement de Dieu et de l'homme. Celui-ci baigne dans la temporalité du fait que ses possibilités et son actualité sont en perpétuelle mouvance. Mais son éternité, ce qui en lui est inchangeable et constant, c'est sa liberté. Taylor définit cette liberté comme étant «... la capacité de prendre quelque ligne d'action» ${ }^{42}$. La détermination elle-même ou la «ligne d'action» (course of action) peut certes varier, mais la capacité qui la soustend est invariable, inchangeable, éternelle.

Il semble bien qu'il s'agisse là d'une capacité de choix pure et simple. Nous regrettons que notre commentateur n'ait pas montré comment s'articule cette conception de l'éternel en l'homme avec l'impératif kierkegaardien, maintes fois proclamé de devenir esprit, de devenir éternel. En d'autres termes, comment notre devoir peut-il être de devenir liberté au sens de capacité de prendre une ligne d'action?

Car il s'agit bien l'à d'une obligation au dire de l'exceptionnel chrétien Anti-Climacus. Devoir déchirant, insiste Kierkegaard dans son Journal de 1851 :

$\mathrm{Si}$, dans notre façon actuelle de vivre, nous sommes vrais devant Dieu, alors nous lui tenons ce langage: je comprends très bien que devenir esprit est réellement l'obligation, mais ne pourriez-vous pas m'accorder un peu d'indulgence? ... J'aimerais tant jouir des choses du monde, et Seigneur Dieu, je ne prétends pasêtre plus fort que je ne suis: je ne suis qu'un enfant ${ }^{43}$.

Du reste, spécialement vers les dernières années de sa vie, le penseur danois exprime avec force et presque impitoyablement les exigences du christianisme, la plus fondamentale étant de devenir esprit. Le Journal de 1854 contient des affirmations percutantes comme celles-ci:

42. "... the capacity to take some course of action". Mark C. Taylor, op.cit. p. 117.

43. Pap. $\mathrm{X}^{3} \mathrm{~A}$ 705. Notre traduction. 
L'esprit est inquiétude; le christianisme est l'inquiétude la plus profonde de l'existence: ainsi en est-il dans le Nouveau Testament. Dans la chrétienté (Cbristenbed) le christianisme (Cbristendom) est repos, de telle sorte que nous puissions jouir de la vie ${ }^{44}$.

Dieu, (selon le christianisme), ne veut pas que les hommes soient en repos: l'esprit est inquiétude ${ }^{45}$.

Devenir chrétien, selon le Nouveau Testament, c'est devenir esprit, devenir esprit, selon le Nouveau Testament, c'est mourir au monde $^{46}$...

La mission de Kierkegaard était précisément de dénoncer cette illusion d'un christianisme facile, commode, statique, comme en fait foi le Point de vue explicatif de mon Oewvre d'Écrivain $^{47}$.

Quant à Anti-Climacus, il est cinglant dans le jugement qu'il porte à l'endroit du faux chrétien:

Tout homme, par la synthèse d'âme et de corps qu'il est, est destiné à être esprit; cet assemblage est sa demeure; mais il préfere habiter le sous-sol, c'est-à-dire les catégories du sensible ${ }^{48} \ldots$

Étant donné l'équivalence des réalités esprit, moi, liberté et éternité, il faut conclure que la liberté est bien pour l'homme un idéal à réaliser, un devoir à remplir. Mais qu'est-ce que cela signifie concrètement?

Une première interprétation possible ${ }^{49}$ serait que l'homme a le devoir et la responsabilité d'acquérir la capacité de choisir sa propre ligne d'action malgré les faux impératifs d'un monde qui vit dans l'illusion. Mais cette capacité, n'est-elle pas là déjà aux premières palpitations de l'esprit qui s'éveille quand surgit l'angoisse? N'est-elle pas, en tant que potentialité, l'apanage de toute synthèse humaine de corps et d'âme? Comment pourrions-nous être dans l'obligation d'acquérir ce que nous avons déjà? Du reste, l'obligation proclamée par l'assesseur Wilhelm de "se choisir

44. Pap. $\mathrm{XI}^{2}$ A 317. Notre traduction.

45. Pap. $\mathrm{XI}^{2} \mathrm{~A}$ 353. Notre traduction.

46. Pap. $\mathrm{XI}^{2} \mathrm{~A}$ 378. Notre traduction. Voir aussi Pour un examen de conscience, $0 . C$. XVIII, p. 77 ss.; S.V. XII, p. 358 ss.; et L'Instant, S.V. XIV, Passim.

47. O.C. XVI, p. 1-102; S.V. XVIII, p. 551-655. Voir aussi là-dessus Cornelio Fabro, «La Missione di Kierkegaard», dans Etbica, VIII (1969), p. 169-180.

48. La Maladie à la Mort, O.C. XVI, p. 201; S.V. XI, 175-176. Voir aussi ibid., O.C. XVI, p. 190 ss.; S.V. XI, p. 165 ss.

49. Nous nous inspirons ici de la pénétrante analyse de Paul Dietrichson, «Kierkegaard's Concept of the Self", dans Inquiry, VIII (1965), p. 1-32. 
soi-même» ou de choisir le choix signifie bien autre chose . . . Ses dires, comme ceux de Vigilius Haufniensis et d'Anti-Climacus, sont difficilement intelligibles sans la présupposition d'une telle capacité dans tout homme, même dans le plus sensuel des esthéticiens. Là où cette capacité n'existe pas, on ne voit pas la possibilité de son surgissement absolu, si ce n'est par une action créatrice. Comme dit l'assesseur:

Si en effet la chose que j'ai choisie n'était pas, mais devenait absolument par le choix, je ne choisirais pas, je créerais; mais je ne me crée pas moi-même, je me choisis ${ }^{50}$.

Une autre interprétation serait: l'homme, du moment qu'il est synthèse même immédiate de corps et d'âme, a en lui-même une capacité de choix qu'il est de son devoir d'actualiser au lieu de la laisser indéfiniment à l'état de rêve ou de pure potentialité. Cette solution à première vue ne manque pas d'attrait: l'esthète $A$ auquel s'en prend l'assesseur n'est-il pas le prototype de celui qui tend à s'abstenir du choix, tout en masquant son abstention elle-même? Quand sonneront les douze coups de minuit et l'heure de lever le masque - pour reprendre la métaphore de l'assesseur -, alors son non-engagement caractéristique sera dévoilé, mais aussi le fait qu'il n'aura jamais pu éluder complètement le choix: s'abstenir de choisir, c'est toujours choisir. Seul le redoublement du choix aura été évité: contrairement à l'éthicien qui choisit le choix, l'esthéticien, lui, choisit de ne pas choisir. Choisir la jouissance, quels que soient son raffinement et la masquarade qu'elle peut comporter, ce n'est pas choisir si ce n'est "au figuré», dit l'assesseur ${ }^{51}$, car

... en proclamant cette intention, on pose toujours une condition qui réside soit en dehors de l'individu, soit en lui, sans être établie par lui ${ }^{52}$.

Il ne fait aucun doute que, pour l'assesseur Wilhelm, l'esthéticien en général et en tant que tel n'est pas engagé dans ce devenir constitutif du moi ou de l'esprit à cause même de son inaptitude à choisir le choix ou à redoubler son choix. Et c'est là également la conviction d'Anti-Climacus et de Kierkegaard lui-même, comme

50. L'Altemative II, O.C. IV, p. 194; S.V. II, p. 232.

51. Ibid., O.C. IV, p. 152; S.V. II, p. 181.

52. Ibid., O.C. IV, p. 164; S.V. II, p. 195. 
nous avons vu. Les chrétiens inauthentiques qu'ils démasquent ne se sont pas vraiment choisis eux-mêmes comme esprits, mais vivent dans la sphère de l'illusion esthétique et de ses choix masqués.

Or si le devoir de devenir liberté n'était rien d'autre que l'obligation d'actualiser une capacité de choix sans plus, l'esthéticien en général qui choisit la jouissance - serait-ce celle que procure l'illusion — répondrait invariablement à cette exigence.

Il est donc nécessaire de préciser que la liberté de choisir ne saurait toucher à ce qui dans l'homme est éternel que dans la mesure où elle orienterait son activité vers la perfection éthique et religieuse.

Mais nous venons de voir que, pour Kierkegaard, une telle capacité de choix est appelée à s'estomper à mesure que l'homme se réalise lui-même comme esprit ou comme liberté. Ou, en d'autres termes, plus la vraie liberté s'accroît en l'homme, et plus sa capacité de choix disparaît pour faire place à une "nécessité intérieure» qui met fin au «tourment» de la décision ${ }^{53}$. La liberté de choix n'est donc pas cet immobile, cet inchangeable qu'est l'éternel en l'homme. Elle apparaît plutôt comme un moyen nécessaire mais provisoire pour y accéder. La nécessité intérieure qui la remplacera, ce sillon d'une ligne d'action concrète creusée par le choix sera la manifestation d'une liberté actualisée.

Concluons. Au lieu d'être une simple possibilité de choix ou l'actualisation même de cette possibilité, la liberté comme tiers synthétisant identique à l'éternel serait plutôt appel ou vocation d'existence. «Qu'est-ce que l'existence?» se demande Johannes Climacus dans le Post-Scriptum. Et il répond:

C'est l'enfant engendré par l'infini et le fini, l'éternel et le temporel, et qui, pour cette raison, est constamment dans l'effort. ${ }^{54}$.

C'est dans l'effort, l'in-quiétude, la douleur de l'enfantement que l'homme doit se constituer lui-mème comme être temporel, c'est-à-dire comme synthèse en voie d'actuation des couples équivalents d'infini et de fini, de possibilité et de nécessité, d'âme et de corps. Accomplir cette synthèse, c'est réaliser un

53. Pap. $\mathrm{X}^{4} \mathrm{~A} 177$.

54. Post-Scriptum aux Miettes pbilosophiques, O.C. X, p. 87, S.V. VII, p. 81 . 
surpassement de l'immédiat dans l'éternel, c'est devenir soimême, c'est exister.

La présence dynamique de l'éternel dans l'homme ne saurait se dire de façon univoque, mais admet tous les degrés possibles de l'analogie: la tâche de se réaliser soi-même n'est jamais achevée.

Au bas de l'échelle, à des degrés divers, se trouve l'esthéticien pour qui l'éternité est appel sans réponse, vain désir de libération. L'assesseur Wilhelm décrit de façon magistrale le tourment masqué de Néron face aux sollicitations de l'éternel:

L'esprit veut toujours surgir, mais sans cesse déçu, il ne peut se faire jour, et Néron le nourrit de la satiété des plaisirs. Alors l'esprit se rassemble en cet homme comme une sombre nuée, sa colère s'appesantit sur l'âme et il se transforme en une angoisse qui ne s'efface pas même au moment de la jouissance ${ }^{55}$.

Les yeux d'une personne sont transparence de l'âme. Mais quand il s'agit de Néron, «derrière ces yeux, l'âme est un amas de ténèbres» ${ }^{56}$. Les caprices essentiellement enfantins de cet homme sont extravagants, cruels, monstrueux parce que l'esprit, sans cesse refoulé, le hante sans arrêt. L'éternel en lui, source de son désarroi, demeure dans un état de pure potentialité. Néron refuse de s'engager dans cette odyssée pénible où sa liberté s'affirmerait, où son moi idéal se rapporterait à son moi réel. Il refuse la compénétration dans l'instant du temporel et de l'éternel. Ou, pour reprendre la formule heureuse de l'assesseur, il refuse de s'enfanter lui-même par l'effort créateur de sa volonté ${ }^{57}$.

L'éclatement de cette sphère où l'esprit est enchaîné ne peut se faire que par un choix redoublé, le choix du choix, ou le choix absolu de soi-même. L'homme se choisit lui-même lorsque, contrairement à Don Juan ou à Néron, il choisit ce devenir pénible qui le constitue esprit en assumant le général.

Mais la libération éthique serait elle-même une illusion si elle arrêtait le mouvement constitutif de l'esprit ou de l'éternel en

55. L'Alternative II, O.C., IV, p. 169; S.V. II, p. 202.

56. Ibid

57. Ibid. O.C. IV, p. 186; S.V. II, p. 222. Le traducteur français des O.C. traduit nisus formativus (employé par Kierkegaard dans le texte danois) par l'expression "effort créateur", mise entre parenthèses. Si l'on accepte cette traduction, il va de soi qu'il ne faut pas comprendre "créateur" dans son sens strict . . . Nous l'avons vu, l'homme n'a pas à se créer lui-même en partant du néant absolu de sa liberté. Nisus formativus pourrait se traduire, quoique moins élégamment, par "effort de réalisation de soi-même". 
recherche de lui-même. La sphère du général devra elle-même éclater pour permettre au moi de gravir un échelon supérieur, celui du religieux. La liberté aura atteint son sommet d'existence quand l'homme se sera choisi lui-même comme chrétien ${ }^{58}$. Mais alors, il sera parvenu à cette in-quiétude profonde, à cette crainte et à ce tremblement dont un chrétien ne peut se départir sans être un hypocrite.

\section{Département de philosophie Université d'Ottawa}

58. Nous avons montré ailleurs que le mouvement d'un stade à l'autre dans l'odyssée du chrétien en devenir est un préparatif humain de l'acte de foi, marque du chrétien authentique. (Voir notre article "L’ambivalence de la réflexion selon Kierkegaard», dans Église et Théologie, mai 1981.) Entre la raison et la foi gît un abîme naturellement infranchissable. Le christianisme n'est pas pour autant irrationnel. "Si je crois, dit Kierkegaard, ni la foi, ni son contenu ne me paraissent assurément absurdes. Non, non. Mais je comprends très bien que pour l'incroyant, les deux le soient . ..» (Pap. $\mathrm{X}^{6}$ B 68). Pour n'avoir pas bien saisi, semble-t-il, la relation que pose Kierkegaard entre foi et raison, Herbert $M$. Garelick le déclare, lui ou tout au moins son pseudonyme Johannes Climacus, inconsciemment anti-chrétien. (Voir The Anti-Christianity of Kierkegaard, The Hague, Martinus Nijhoff 1965.) Notons bien que son ouvrage porte exclusivement sur le Post-Scriptum et que lauteur ne décide pas si Kierkegaard est anti-chrétien comme l'est Climacus. Le titre est donc fort ambigu, Kierkegaard se situant dialectiquement entre ses deux pseudonymes Climacus, le nonchrétien, et Anti-Climacus, le chrétien extraordinaire. (Pap. $\mathrm{X}^{2}$ A 195). La thèse de Garelick est que Johannes Climacus non seulement n'est pas chrétien, mais ne comprend pas ce qu'est le christianisme. En effet, après l'avoir défini comme irrationnel, il en fait non pas une fin en soi, mais un moyen d'accéder à quelque chose d'autre: le bonheur éternel. Le christianisme est ainsi doublement dénaturé: l'irrationnel qu'il est devient raisonnable, et l'absolu qu'il est devient subordonné à une finalité autre. D'où anti-christianisme qui pourtait bien être celui de Kierkegaard lui-même, puisque, sur la page couverture du Post-Scriptum, il se dit éditeur de l'ouvrage de Johannes Climacus. Garelick remarque: «As Kierkegaard moves his name closer to actual authorship, his works come closer to his final position .... (p. 8). L'ouvrage de Garelick ne nous a guère convaincu quant à la position exacte de Climacus sur l'irrationalité de l'acte de foi. D'autre part, Je pseudonyme le plus proche de la pensée de Kierkegaard est vraisemblablement Anti-Climacus. (Voir là-dessus l'étude d'Alastair McKinnon, «Kierkegaard's Pseudonyms: A New Hierarchy", dans American Philosopbical Quarterly, Vol. VI, No 2, April 1969, p. 116-126). Et nombre d'extraits des Papirer montrent qu'il est loin de l'anti-christianisme dont parle Garelick. (Voir là-dessus C. Fabro, «Foi et Raison dans l'oeuvre de Kierkegaard», dans Revue des sciences philosophiques et théologiques, 32 (1948), p. 169-206.) 